\title{
Incommmensurability and unconventional superconductor to insulator transition in the Hubbard model with bond-charge interaction
}

\author{
A. A. Aligia, ${ }^{1}$ A. Anfossi,${ }^{2,3}$ L. Arrachea, ${ }^{4,3}$ C. Degli Esposti Boschi,${ }^{5}$ A. \\ O. Dobry, ${ }^{6}$ C. Gazza, ${ }^{6}$ A. Montorsi, ${ }^{2}$ F. Ortolani, ${ }^{7}$ and M. E. Torio ${ }^{6}$ \\ ${ }^{1}$ Comisión Nacional de Energía Atómica, Centro Atómico Bariloche and Instituto Balseiro, 8400 S.C. de Bariloche, Argentina \\ ${ }^{2}$ Dipartimento di Fisica del Politecnico and CNISM, \\ corso Duca degli Abruzzi 24, I-10129, Torino, Italy \\ ${ }^{3}$ BIFI, Universidad de Zaragoza, Corona de Aragón 42, 5009 Zaragoza, Spain \\ ${ }^{4}$ Departamento de Física de la Materia Condensada, Universidad de Zaragoza, 5009 Zaragoza \\ ${ }^{5}$ Unità CNISM and Dipartimento di Fisica dell'Università di Bologna, viale Berti-Pichat 6/2, I-40127, Bologna, Italy \\ ${ }^{6}$ Instituto de Física Rosario, CONICET-UNR, Bv. 27 de Febrero 210 bis, 2000 Rosario, Argentina. \\ ${ }^{7}$ Dipartimento di Fisica dell'Università di Bologna and INFN, viale Berti-Pichat 6/2, I-40127, Bologna, Italy
}

(Dated: November 1, 2018)

\begin{abstract}
We determine the quantum phase diagram of the one-dimensional Hubbard model with bondcharge interaction $X$ in addition to the usual Coulomb repulsion $U>0$ at half-filling. For large enough $X<t$ the model shows three phases. For large $U$ the system is in the spin-density wave phase as in the usual Hubbard model. As $U$ decreases, there is first a spin transition to a spontaneously dimerized bond-ordered wave phase and then a charge transition to a novel phase in which the dominant correlations at large distances correspond to an incommensurate singlet superconductor.
\end{abstract}

PACS numbers: 71.10.Fd,71.10.Hf,71.10.Pm,71.30.+h

The Hubbard model has been originally proposed to describe the effect of the Coulomb interaction in transition metals, which usually contain localized orbitals. Other real compounds containing more extended orbitals cannot in general be properly described by this simple Hamiltonian. Well-known examples are several quasione-dimensional (1D) materials that have been recently investigated [1], which exhibit a variety of phases that cannot be explained with the usual Hubbard model. Additional interactions should be included. A natural interaction that arises in systems with extended orbitals is the bond-charge interaction $X$ [2]. In fact, it is natural to assume that the charge in the bond affects screening and the effective potential acting on valence electrons, and therefore the extension of the Wannier orbitals and the hopping between them should vary with the charge.

This leads to the $U-X$ Hamiltonian:

$$
\begin{aligned}
H= & -t \sum_{\sigma=\uparrow, \downarrow,\langle i j\rangle}\left(c_{i \sigma}^{\dagger} c_{j \sigma}+\text { H.c. }\right)+U \sum_{i} n_{i \uparrow} n_{i \downarrow} \\
& -X \sum_{\sigma,\langle i j\rangle}\left(c_{i \sigma}^{\dagger} c_{j \sigma}+\text { H.c. }\right)\left(n_{i-\sigma}+n_{j-\sigma}\right) .
\end{aligned}
$$

This model has been studied in two dimensions, motivated by a theory of hole superconductivity [3]. A modified version of it has been derived as an effective model for the cuprates and shows enhanced $d$-wave superconducting correlations [4]. Recently, this model has been paramount to broader audiences, and its relevance has been discussed in the context of mesoscopic transport [5] and quantum information [6, 7].

In 1D, there are bosonization [8, 9] and numerical [9] results available. However, at half-filling, the effect of $X$ disappears in the standard bosonization treatment and a behavior different from the usual Hubbard model was not expected in these studies. For $X=t$, an exact solution is available [10]. In this case the ground state is highly degenerate: the transition to a metallic state takes place at $U_{c}=4 t>0$, but the response of the system to an applied magnetic flux indicates that it is not superconducting 11]. In view of the previous studies, the recent evidence of an insulator-metal transition driven by $X<t$ at finite $U_{c}>0$ at half-filling comes as a surprise [12]. The nature of the metallic phase and the character of the transition have not been fully elucidated, though the possibility of superconductivity has been suggested.

In this Letter we employ several analytical and numerical techniques to calculate accurately the phase diagram of the model at half-filling in $1 \mathrm{D}$ and to determine the nature of each phase. We establish that the insulator-metal transition is of commensurate-incommensurate (CIC) type to a phase with dominating singlet superconducting (SS) correlations. Remarkably, unlike other CIC transitions [13, 14], it is not driven by one-body effects like chemical potential or the emergence of more than two Fermi points in the noninteracting dispersion relation, but by strong correlations induced by large enough $X$. In addition, we unveil that inside the insulating phase there is a spin transition separating the expected spin-density wave (SDW) for $U>U_{s}$ from a spontaneously dimerized bond-ordered wave (BOW) phase for $U_{c}<U<U_{s}$. This transition is of Kosterlitz-Thouless (KT) type and a spin gap opens in the BOW phase.

The nature of each phase and the qualitative aspects of the phase diagram can be understood by a weak coupling bosonization analysis provided it includes vertex corrections of second order in $X$ to the coupling constants and 
one term of order $a^{2}$ in the bosonization of the bondcharge interaction as described below, where $a$ is the lattice constant. A bosonized version of (1) is given by the following Hamiltonian density:

$$
\begin{aligned}
\mathcal{H} & =\mathcal{H}_{\sigma}^{0}+\mathcal{H}_{\rho}^{0}+\frac{2 g_{1 \perp}}{(2 \pi \alpha)^{2}} \cos \left(\sqrt{8} \phi_{\sigma}\right)-\frac{2 g_{3 \perp}}{(2 \pi \alpha)^{2}} \cos \left(\sqrt{8} \phi_{\rho}\right) \\
& +\frac{2 g_{\sigma \rho}}{(2 \pi \alpha)^{2}} \cos \left(\sqrt{8} \phi_{\sigma}\right) \partial_{x} \phi_{\rho}
\end{aligned}
$$

where $\mathcal{H}_{\sigma}^{0}$ and $\mathcal{H}_{\rho}^{0}$ are the usual known quadratic forms and $\alpha$ is a short range cutoff in the bosonization procedure. The first line of (2) has the structure of the previously studied bosonized theory [8], which corresponds to two decoupled sine-Gordon field theories, one for the spin $\left(\phi_{\sigma}\right)$ and the other for the charge $\left(\phi_{\rho}\right)$. In order to take into account the effect of the bond-charge interaction on the phase diagram of the system, we included vertex corrections of second order in $X$ in the definition of the the coupling constants $g_{i}$, due to virtual processes involving states far from the Fermi energy [15]. In addition, we took into account the usually neglected $g_{\sigma \rho}$ term that couples spin and charge degrees of freedom. The latter is $\propto a^{2}$. It arises including spatial derivatives of the fermionic fields in the representation of (1) in terms of a low energy field theory. All of these terms have naive scaling dimension 3 and are usually neglected. However, one term that bosonize as the second line of (2) becomes relevant for large enough $X$ and provides a mechanism for an incommensurate transition, as discussed below.

Explicitly, the effective parameters read $g_{1 \perp}=g_{2 \perp}=$ $\left(U-\frac{8 X^{2}}{\pi(t-X)}\right) a$ and $g_{\sigma \rho}=\sqrt{2} a^{2} X$. The forward and umklapp processes are the same as in the Hubbard model, $g_{3 \perp}=g_{4 \perp}=U a$. The Luttinger liquid parameters $\left(K_{\rho}\right.$ and $\left.K_{\sigma}\right)$ and the charge and spin wave velocity $\left(u_{\rho}\right.$ and $u_{\sigma}$ ) in terms of $g_{i}$ are given by known expressions [16]. Neglecting the $g_{\sigma \rho}$ term, the renormalization-group $(\mathrm{RG})$ flow diagrams are of KT type. A spin gap opens when $g_{1 \perp}<0$, i.e., when the flow of RG, which takes place on the separatrix of the KT diagram due to spin $\mathrm{SU}(2)$ symmetry, goes to strong coupling. Therefore, the spin gapped phase appears when $U<U_{s}=\frac{8 X^{2}}{\pi(t-X)}$. As for the behavior of the charge modes, a gap opens when the $g_{3} \perp$ term becomes relevant. The charge gapped phase takes place for $U>U_{c}$, with $U_{c}<U_{s}$. The $g_{\sigma \rho}$ term becomes relevant for $K_{\sigma}<1 / 2(X>0.6 t$ for $U=0)$. In the spin gapped phase the $\cos \left(\sqrt{8} \phi_{\sigma}\right)$ is frozen at its mean value. This term could be interpreted as a chemical potential $\left[\mu=\frac{2 g_{\sigma \rho}}{(2 \pi \alpha)^{2}}\left\langle\cos \left(\sqrt{8} \phi_{\sigma}\right)\right\rangle\right]$ times a charge density operator. The effects of such a term are known [16]. If we start the analysis from a situation where there is also a charge gap $\left(\Delta_{c}\right)$ smaller than the spin one $\left(\Delta_{s}\right)$, and we then increase the value of $X$, the effect of this term is to close $\Delta_{c}$, leading to a metallic phase when $\mu>\Delta_{c}$. The effective Fermi level is shifted with respect to the original one and the system develops incommen-

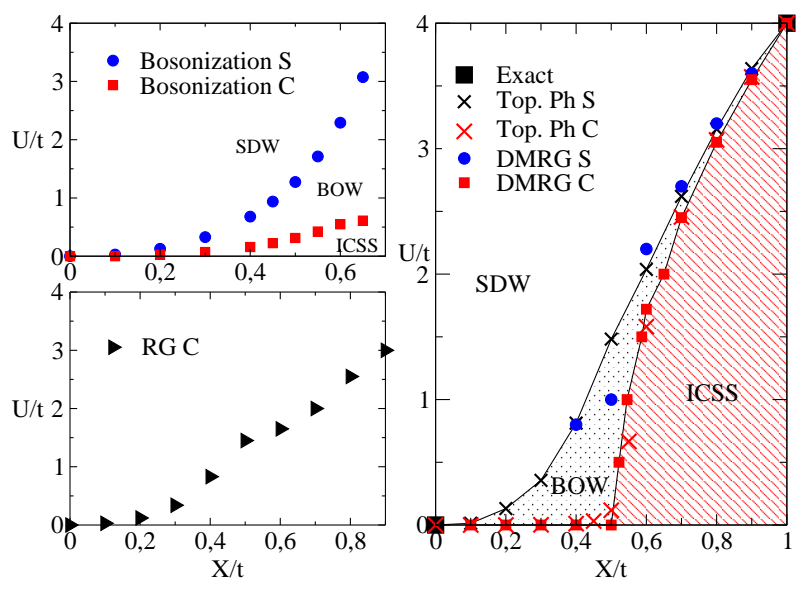

Figure 1: (Color online). Phase diagram. Left: Bosonization (top), and real space renormalization-group (bottom) predictions. Right: Numerical results as obtained by DMRG (circles-squares) and topological phase (crosses) methods.

surate correlations. A numerical analysis discussed below shows that the system has dominant SS correlations. Thus, this phase can be characterized as incommensurate singlet superconducting (ICSS).

For a qualitative localization of the boundary transition line between the insulator and the ICSS phase, we have implemented a procedure as follows: (i) We start from a parameter regime where the spin gap is open. (ii) We follow the RG flow up to a length scale where $\left|g_{1 \perp}\right| /\left(\pi U_{s}\right) \mid \sim 1$. (iii) At this point the $g_{\sigma \rho}$ term is decoupled by a mean field approach similar to that used by Nersesyan et al. to show incommensurability in the anisotropic zigzag chain [17]. The value of $\left\langle\cos \left(\sqrt{8} \phi_{\sigma}\right)\right\rangle$ is exactly obtained at the LE point $\left(K_{\sigma}=1 / 2\right)$. (iv) For vanishing $g_{\sigma \rho}, \Delta_{c}$ is obtained by rescaling the problem to the LE point of the charge sector, by using the RG equations of the sine-Gordon theory. (v) The CIC transition takes place when $\frac{2 g_{\sigma \rho}}{(2 \pi \alpha)^{2}}\left\langle\cos \left(\sqrt{8} \phi_{\sigma}\right)\right\rangle=\Delta_{c}$ [16]. In the top left panel of Fig. 1 we show the phase diagram of the model predicted by this approach. For each value of $X$, there are two transition points $U_{c}$ and $U_{s}$ corresponding to the charge and spin transition, respectively. Each phase is characterized by the gapped modes and the relevant order parameter. For $U>U_{c}$ the system is an insulator. For $U>U_{s}$, the slowest decaying correlation functions are the spin-spin ones. The system is in a SDW phase. For $U_{c}<U<U_{s}$ a fully gapped (spin and charge) phase is developed. The fields $\phi_{\sigma}$ and $\phi_{\rho}$ are located at the minimum of the potential, and the translation symmetry is spontaneously broken. The BOW parameter, defined below, acquires a nonzero value. For $U<U_{c}$ the charge gap closes and the dominant correlations at large distances are the SS ones. While the nature of each phase has been identified, the phase boundaries predicted by bosonization are not quantitatively valid, particularly 
for large values of the interactions. In the right panel of Fig. 1 we show the phase diagram of the model, as obtained by accurate numerical techniques. One of them, used to determine the charge transition line, consists in studying singularities of single-site entanglement [12] by means of density-matrix renormalization group (DMRG) [18]. Another method is based on topological numbers, or jumps of Berry phases [19], which was successfully applied to a similar model [8] (b). The value of $U_{c}\left(U_{s}\right)$ is determined in this case by the jump of the charge (spin) Berry phase. The corresponding values of $U_{c}$ and $U_{s}$ in systems up to $L=14$ sites, extrapolated to the thermodynamic limit using a parabola in $1 / L^{2}$, are also shown in Fig. 1]

DMRG evaluations of $\Delta_{c}$ and $\Delta_{s}$ confirm these predictions. The charge gap was calculated in 12 from the definition $2 \Delta_{c}=E_{0}(N+2)+E_{0}(N-2)-2 E_{0}(N)$, $E_{0}(N)$ being the ground-state energy of the chain with $N$ particles. Similarly, the spin gap is here determined through $\Delta_{s}=E_{0}\left(S_{z}=1\right)-E_{0}\left(S_{z}=0\right)$, being $E_{0}\left(S_{z}\right)$, the ground-state energy of the half-filled system within the subspace with a given total $S_{z}$. We can see in Fig. 1 that the closing of $\Delta_{c}, \Delta_{s}$ do not take place simultaneously for small $U$ and $X$. The critical lines for the closing of both gaps obtained by extrapolations to the thermodynamic limit are in reasonable quantitative agreement with the ones determined by the method of the topological phases.

We have verified that the spin transition is of $\mathrm{KT}$ type, calculating the scaling dimensions of the singlet and triplet operators as described in [19]. In order to identify the universality class of the charge transition, we employed the finite-size crossing method [20]. The study of the dependence of $\left\langle n_{i \uparrow} n_{i \downarrow}\right\rangle=\partial e_{L} / \partial U$ on the size $L\left(e_{L}\right.$ being the ground-state energy density) provides a location of the critical points in agreement with the methods discussed above. In addition, the divergence that develops $\partial e_{L}^{2} / \partial U^{2}$ with increasing $L$ indicates that the gap exponent $\nu$ remains close to $1 / 2$ (the value that can be computed exactly at the point $X=t$ ) for $X / t=0.6,0.7,0.8$ with a possible increase for $X / t \rightarrow 0.5$; below this point, our numerical analysis suggests that the charge transition becomes of KT type, with " $\nu=\infty$ ". The estimate $\nu=1 / 2$ relies upon the assumption that the dynamic exponent $\zeta$ (through which gap and correlation length $\xi$ are related, $\Delta_{c} \propto \xi^{\zeta}$ ) is still $\zeta=2$, as in the exactly solvable case $X=t$ [7]. As already noted in [12], the behavior of $\Delta_{c} \propto L^{-2}$ along the transition line is consistent with this exponent. We stress that such feature is in agreement with the CIC character of the metal-insulator transition [16]. Instead, within the metallic phase, the finite-size scaling suggests $\Delta_{c} \propto L^{-1}$, although the data are rather noisy due to incommmensurability.

In Fig. 2 we show numerical results supporting the incommensurate character of the metallic phase. We report the density distributions in real space for the local charge

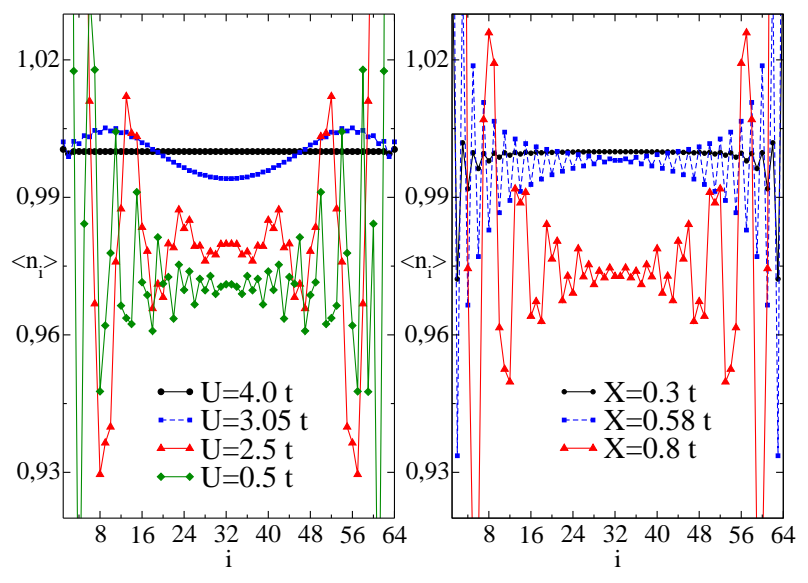

Figure 2: (Color online). Charge distribution $\left\langle n_{i}\right\rangle$ evaluated by DMRG. Left: $X=0.8 t$. Right: $U=1.5 t$.

density $n_{i}=n_{i \uparrow}+n_{i \downarrow}$ in the ground state in an open chain with $L=64$ sites. The incommensurate character of the metallic phase manifests itself also in the behavior of the charge and spin correlation functions, whose corresponding structure factors show peaks away from the commensurate reciprocal vector $q=\pi$ (not shown). The left panel of Fig. 2 corresponds to $X=0.8 t$ as $U$ is varied. The behavior is similar to the one observed within the incommensurate phase of the Hubbard model including next-nearest-neighbor hopping $\left(t-t^{\prime}-U\right.$ model $)[13]$. For $U>U_{c}=3.05 t$, the commensurate charge distribution characterizing the insulating phase is reached within a few lattice sites from the edge. The insulator-metal transition shows up via the appearance of incommensurate modulations in the charge distribution, whose wavelength increases within the metallic phase. The right part of the figure shows the results obtained by varying $X$ at $U=1.5 t$. Interestingly, a first modulation appears already for $X_{s}<X<X_{c}\left(X_{s} \approx 0.5 t\right.$, and $\left.X_{c} \approx 0.6 t\right)$. Again, for $X>X_{c}$ further incommensurate modulations appear in the LE phase.

Within the charge sector $U<U_{c}$, the dominating correlations at large distance are superconducting pair-pair ones if the correlation exponent $K_{\rho}>1$ or charge-charge ones otherwise. We calculated $K_{\rho}$ employing the methodology described in 9]. This study casts extrapolated values $K_{\rho} \sim 1.3$ for $U=0$ and $X=0.8 t$. To provide stronger evidence for the SS character of the incommensurate phase, we have calculated on-site pairing correlations $\left\langle P_{i}^{\dagger} P_{j}\right\rangle$ with $P_{i}=c_{i \uparrow}^{\dagger} c_{i \downarrow}^{\dagger}$ and charge-charge correlations $\left|\left\langle n_{i} n_{j}\right\rangle-\left\langle n_{i}\right\rangle\left\langle n_{j}\right\rangle\right|$ in an open chain with 100 sites and using the sites 30 to 70 to avoid boundary effects. The results are displayed in Fig. 3. A fitting of the pairing correlations at distances between 8 and 40 sites gives $K_{\rho}=1.32 \pm 0.01$. This value is also consistent with the long distance behavior of the charge-charge correlations. The inset also shows the tendency of the system to show the anomalous flux quantization characteristic of super- 


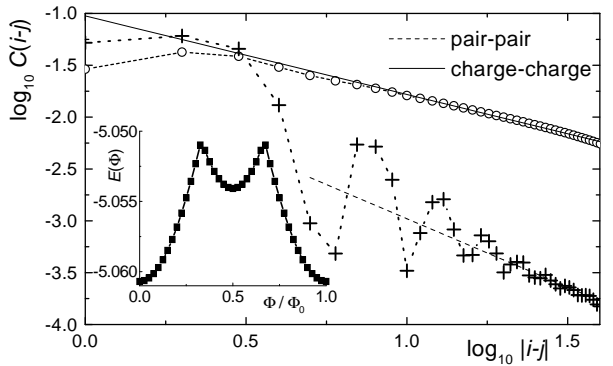

Figure 3: Pair-pair and charge-charge correlation functions for $U=t$ and $X=0.8 t$. Full (dashed) line corresponds to a power law with exponent $1 / K_{\rho}\left(K_{\rho}\right)$. The inset shows the ground state energy as a function of an applied magnetic flux.

conductivity [1], which is more pronounced as the size of the system increases.

An additional argument suggesting superconducting correlations within this phase is provided by the real space renormalization-group method, used before for the standard Hubbard model [21]. Different from that case, the recursive equations for the renormalized parameters in the positive $U$ regime, depending on $X$ and $U$, exhibit three different fixed points for the $n$th step renormalized Coulomb interaction $U^{(n)}$ in the large $n$ limit: $U^{(n)}>0$ for $U>U_{r c}, U^{(n)}=0$ for $U=U_{r c}$, and $U^{(n)}<0$ for $U<U_{r c}$. In the latter case, the effective Coulomb interaction becomes attractive. In the bottom left insert of Fig. $10 U_{r c}$ obtained in this way is reported.

To support the bosonization predictions, which characterize the intermediate phase as a BOW, we have evaluated with DMRG the BOW order parameter $O_{B O W}=$ $\left[\sum_{i, \sigma}(-1)^{i}\left\langle c_{i+1 \sigma}^{\dagger} c_{i \sigma}+H . c.\right\rangle\right] /(L-1)$ in chains with open boundary conditions, following the same procedure as Manmana et al. for the ionic Hubbard model 22] in chains up to 400 sites. In spite of the large systems used, finite-size effects are still important and do not allow an accurate extrapolation. In any case, the qualitative behavior of our results (not shown) is similar to that found by Manmana et al. showing a clear maximum inside the BOW phase, an abrupt fall for $U \sim U_{c}$ as the system enters the SS phase and a slower decay for larger $U \sim U_{s}$, which for finite systems extends inside the SDW phase.

To conclude, we have presented compelling evidence, based on bosonization as well as on other analytical and numerical techniques, of the existence of a narrow bondordered wave phase and a transition to an unconventional incommensurate metallic one with dominant singlet superconducting correlations in the phase diagram of the $U-X$ model. The appearance of superconductivity in a model with repulsive on-site interactions at half filling, and of incommensurate correlations induced by interaction are both unusual features. Their emergence can be understood from the structure of the exactly solvable case $X=t$. There the number $N_{d}$ of doubly occupied sites (doublons) becomes a conserved quantity; holes and doublons play an identical role regarding the kinetic energy $\epsilon\left(k_{F}\right)$, which can be mapped into that of a spinless fermion system, with Fermi momentum $k_{F}$. The competition of $\epsilon\left(k_{F}\right)$ and $U N_{d}$ fixes the Fermi level of the resulting effective model. The presence of doublons in the ground state $(U<4 t)$ simultaneously drives the spinless fermions away from half-filling $\left(k_{F} \neq \pi\right)$, and switches on the doublons role in the kinetic energy. The latter ceases to be identical to that of holes as soon as $X \neq t$, generating incommensurability within the system. Moreover superconducting correlations can dominate away from half-filling [8]. Thus, a nonvanishing number of doublons provides the scenario for both incommensurability and superconductivity for $X \lesssim t$.

We thank D. Cabra for useful discussions. We acknowledge support from PICT's No. 03-11609, No. 03-12742, and No. 05-33775 of ANPCyT and PIP's No. 5254 and No. 5306 of CONICET, Argentina, No. FIS200608533-C03-02, and the "Ramon y Cajal" program from MCEyC of Spain, Angelo Della Riccia Foundation, and PRIN 2005021773 Italy.

[1] Burbonais, Science 281, 1155 (1998); H. Kishida et al., Nature (London) 405, 929 (2000).

[2] J. T. Gammel and D. K. Campbell, Phys. Rev. B 60, 71 (1988); Y. Z. Zhang, ibid. 92, 246404 (2004); R. Strack and D. Volhardt, Phys. Rev. Lett. 70, 2637 (1993).

[3] J. E. Hirsch, Physica (Amsterdam) 158C, 326 (1989); J. E. Hirsch and F. Marsiglio, Phys. Rev. B 39, 11515 (1989).

[4] L. Arrachea and A. A. Aligia, Phys. Rev. B 59, 1333 (1999); ibid. 61, 9686 (2000).

[5] A. Hübsch et al., Phys. Rev. Lett. 96, 196401 (2006).

[6] A. Anfossi et al. Phys. Rev. Lett. 95, 056402 (2005).

[7] A. Anfossi, P. Giorda, and A. Montorsi, Phys. Rev. B 75, 165106 (2007).

[8] G. I. Japaridze and E. Müller-Hartman, Ann. Phys. (Leipzig) 506, 163 (1994); A. A. Aligia and L. Arrachea, Phys. Rev. B 60, 15332 (1999), and references therein.

[9] L. Arrachea et al., Phys. Rev. B 50, 16044 (1994).

[10] L. Arrachea and A. A. Aligia, Phys. Rev. Lett. 73, 2240 (1994); J. de Boer, V. E. Korepin, and A. Schadschneider, ibid. 74, 789 (1995).

[11] L. Arrachea, A. A. Aligia and E. Gagliano, Phys. Rev. Lett. 76, 4396 (1996).

[12] A. Anfossi et al., Phys. Rev. B 73, 085113 (2006).

[13] G. I. Japaridze, R. M. Noack, and D. Baeriswyl, Phys. Rev. B 76, 115118 (2007).

[14] I. N. Karnaukhov, Phys. Rev. B 66, 092304 (2002).

[15] M. Tsuchiizu and A. Furusaki, Phys. Rev. B 69, 035103 (2004).

[16] T. Giamarchi, Quantum Physics in One Dimension (Oxford University Press, Oxford, U.K., 2004).

[17] A. A. Nersesyan, A. O. Gogolin, and F.H.L. E $\beta$ ler, Phys. 
Rev. Lett. 81, 910 (1998).

[18] S. R. White, Phys. Rev. Lett. 69, 2863 (1992); K. Hallberg, Adv. Phys. 55, 477 (2006).

[19] M. E. Torio et al., Phys. Rev. B 73, 115109 (2006), and references therein.
[20] L. Campos Venuti et al., Phys. Rev. A 73, 010303(R) (2006).

[21] J. E. Hirsch, Phys. Rev. B 22, 5259 (1980).

[22] S. R. Manmana et al., Phys. Rev. B 70, 155115 (2004). 